\title{
Aspectos nutricionais da variação genética em progênies de Myracrodruon urundeuva Fr. All. sob diferentes condições de cultivo
}

\author{
Simone Aparecida de Oliveira ${ }^{(1)}$ \\ Mario Luiz Teixeira de Moraes ${ }^{(2)}$ \\ SAlatí́r BuZetti ${ }^{(3)}$
}

\begin{abstract}
RESUMO
Sementes de aroeira foram obtidas a partir de 30 árvores de polinização livre, localizadas na Estação Ecológica do Instituto Florestal em Paulo de Faria - SP, em setembro de 1996. Foram instalados dois testes de progênies de aroeira (consorciado com mutambo e angico do campo - Exp 1 e homogêneo - Exp 2), em março de 1997, na Fazenda de Ensino e Pesquisa da FEIS/UNESP, em Selvíria - MS. O delineamento experimental utilizado foram os blocos casualizados, tanto no experimento consorciado como no homogêneo. Os caracteres nutricionais avaliados foram o conteúdo de: N, P, K, $\mathrm{Ca}, \mathrm{Mg}$ e S. As estimativas de parâmetros genéticos foram obtidas em nível de média de parcelas. A herdabilidade, aos dois anos, variou de 0,05 (para o Mg) a 0,63 (para o N). Foi encontrada variabilidade genética para o teor de nutrientes e a característica mais indicada para um processo de seleção seria o conteúdo de N.
\end{abstract}

Palavras-chave: aroeira, sistema de plantio, teste de progênies, parâmetros genéticos, teor de nutrientes

\section{ABSTRACT}

Nutrient aspects of genetic variation on progeny of Myracrodruon urundeuva Fr. All. under different condition of crop. Aroeira seeds were obtained from 30 trees of open pollination, located in the Estação Ecológica do Instituto Florestal in Paulo de Faria - SP, in September, 1996. Two tests of Aroeira progeny were conducted (mutambo and angico together - Exp 1 and homogeneous - Exp 2), in March, 1997, at experimental station of FEIS/UNESP, in Selvíria - MS. A randomized completely block design, to both experiments was used. The nutrient content evaluated was: N, P, K, Ca, $\mathrm{Mg}$, and $\mathrm{S}$. The genetic estimates achieved to mean plot level and herdability on 2 year old trees was 0,05 to $\mathrm{Mg}$ and 0,63 to $\mathrm{N}$. There was genetic variability

${ }^{(1)}$ Bolsista da FAPESP - IC e Pós-graduanda em Agronomia FEIS/UNESP - Av. Brasil Centro, 56 Ilha Solteira - SP. CEP - 15.385-000. E-mail: simoneap@agr.feis.unesp.br

${ }^{(2)}$ Professor Dr. do Departamento de Fitotecnia, Economia e Sociologia Rural - FEIS/UNESP E- mail: teixeira@agr.feis.unesp.br

${ }^{(3)}$ Professor Dr. do Departamento de Ciência do Solo e Engenharia Rural - FEIS/UNESP - E- mail: $\underline{\text { sbuzetti@agr.feis.unesp.br }}$ 
to nutrient content and $\mathrm{N}$ was the better characteristic to selection process.

Key words: Aroeira, plantation system, progeny test, genetic parameters, nutrient content

\section{INTRODUÇÃO}

As espécies arbóreas são uma das principais fontes geradoras de energia desde a antigüidade, sendo também utilizadas no setor madeireiro, como fonte medicinal e para alimentação (forrageira, quando nova).

Entre as espécies arbóreas destacam-se as da família Anacardiaceae, especificamente a aroeira (Myracrodruon urundeuva Fr. All.), a qual produz madeira de alta densidade, de longa durabilidade. De sua casca se extrai o tanino, para uso medicinal as folhas servem como forrageira e, pela beleza de sua copa, esta planta serve como ornamental, além de ser usada em reflorestamento ambiental e em sistemas agroflorestais.

O sucesso dos programas de reflorestamento depende da qualidade das mudas e do tempo de sua formação, visando a resistência às condições ambientais adversas e a redução no custo de formação de mudas, respectivamente. Neste contexto, o fornecimento equilibrado de nutrientes para as plantas é um fator essencial (MENDONÇA et al., 1998).

Segundo MALAVOLTA et al. (1989), a composição mineral da folha, ou o teor dos elementos nela encontrados, é consequiência dos fatores que atuaram e, às vezes, interagiram até o momento em que o órgão foi colhido para análise (ou até mesmo depois da tomada da amostra). Em um estudo sobre variação genética no teor de minerais ( $\mathrm{N}, \mathrm{P}, \mathrm{K}, \mathrm{Ca}, \mathrm{Mg}, \mathrm{Na}$ ), proteína crua e carboidrato estrutural em 31 procedências de Prosopis cineraria (L.) Druce, na Índia, ARYA et al. (1995) encontraram diferenças significativas entre as mesmas, nas características analisadas.

No que se refere aos teores foliares de macronutrientes, a literatura ainda é escassa, devendo ser encorajadas mais pesquisas nesta área. Neste sentido, MENDONÇA et al. (1998) verificaram que os teores foliares nas plantas associados ao melhor desenvolvimento de aroeira foram: $\mathrm{N}=23,6 \mathrm{~g} / \mathrm{kg}$; $\mathrm{P}=1,0 \mathrm{~g} / \mathrm{kg} ; \mathrm{K}=13,6 \mathrm{~g} / \mathrm{kg} ; \mathrm{Ca}=14,8 \mathrm{~g} / \mathrm{kg} ; \mathrm{Mg}=2,2 \mathrm{~g} / \mathrm{kg} ; \mathrm{B}=18,35 \mathrm{mg} / \mathrm{kg}$ e $\mathrm{Zn}=18,57 \mathrm{mg} / \mathrm{kg}$. BARBOSA (1994) verificou que, nas mudas de aroeira que não receberam $\mathrm{P}$ ou $\mathrm{Zn}$, os teores foliares foram de: $\mathrm{N}=27,6 \mathrm{~g} / \mathrm{kg} ; \mathrm{P}=0,8 \mathrm{~g} / \mathrm{kg}$; $\mathrm{K}=10,7 \mathrm{~g} / \mathrm{kg} ; \mathrm{Ca}=6,1 \mathrm{~g} / \mathrm{kg} ; \mathrm{Mg}=3,5 \mathrm{~g} / \mathrm{kg}$ e $\mathrm{S}=2,0 \mathrm{~g} / \mathrm{kg}$. FREITAS (1999) encontrou os seguintes teores foliares: 25,0 e $26,7 \mathrm{~g} / \mathrm{kg} \mathrm{N} ; 1,8$ e $1,8 \mathrm{~g} / \mathrm{kg} \mathrm{P}$; 13,2 e 14,8 g/kg K; 6,1 e 7,1 g/kg Ca; 2,8 e 3,4 g/kg Mg; 1,2 e 1,2 g/kg S, para aroeira em plantio homogêneo e consorciado com candiúba, respectivamente. MORAES (1992), estudando duas populações de aroeira, aos 3,5 anos de idade, encontrou teores de nutrientes nas folhas de: 18,92 e 19,06 g/kg N, 1,18 e 1,28 $\mathrm{g} / \mathrm{kg} \mathrm{P}, 8,03$ e 7,66 g/kg K, 6,73 e 7,52 g/kg Ca, 2,15 e 2,40 g/kg Mg, 0,73 e 
$0,85 \mathrm{~g} / \mathrm{kg} \mathrm{S}$, para as populações de Bauru - SP e Selvíria - MS, respectivamente.

O conhecimento do nível de variação genética e da sua distribuição, entre e dentro das populações de plantas das espécies florestais, assume importância na escolha das estratégias de melhoramento a serem adotadas, visando maximizar os ganhos genéticos através dos ciclos de seleção. Esse conhecimento é fundamental, também, para o manejo de populações naturais visando a conservação genética (DIAS \& KAGEYAMA, 1991).

O presente trabalho teve como objetivo estimar a variabilidade genética de uma população natural de aroeira, conduzida em dois sistemas de plantio, para as principais características nutricionais.

\section{MATERIAL E MÉTODOS}

As sementes de aroeira utilizadas neste trabalho foram obtidas a partir de 30 árvores de polinização livre, localizadas na Estação Ecológica do Instituto Florestal em Paulo de Faria - SP, sendo coletadas em setembro de 1996. Sementes de mutambo e angico do campo foram coletadas na região de Ilha Solteira - SP. As mudas das espécies florestais que fazem parte do experimento foram produzidas em tubetes no viveiro da Companhia Energética de São Paulo - CESP em Ilha Solteira - SP.

Foram instalados dois testes de progênie, o primeiro foi sombreado (aroeira consorciada com mutambo e angico do campo - EXP1) e, o segundo a pleno sol (plantio homogêneo - EXP2), em março de 1997, no município de Selvíria - MS. O delineamento experimental utilizado foi o de blocos casualizados, em ambos os experimentos. O plantio foi efetuado no espaçamento de $3,0 \mathrm{~m}$ x 3,0 m no experimento consorciado e de $3,0 \mathrm{~m} \times 1,5 \mathrm{~m}$ no experimento a pleno sol, com 30 tratamentos (progênies) e 3 repetições. As parcelas forma instaladas na forma linear com 10 plantas. No experimento sombreado, cada planta de aroeira ficou entre uma planta de angico e outra de mutambo, a uma distância de $1,5 \mathrm{~m}$, na mesma linha de plantio.

Os caracteres nutricionais avaliados foram os teores de $\mathrm{N}, \mathrm{P}, \mathrm{K}, \mathrm{Ca}$, $\mathrm{Mg}$ e $\mathrm{S}$ nas folhas. As avaliações foram realizadas aos 26 meses de idade das plantas.

As folhas foram coletadas de todas as árvores, de cada parcela, constituindo, assim, amostras compostas de cada parcela. Cada progênie foi representada por 3 amostras compostas, oriundas das respectivas repetições. Esse procedimento foi realizado nos dois experimentos (consorciado e homogêneo).

O local de amostragem foi padronizado para todas as árvores, no terço médio da copa, em folhas de mesma idade (intermediárias no ramo escolhido), nos 4 pontos cardeais, retirando-se 4 folhas por planta. As amostras foram 
identificadas e colocadas em sacos de papel e o material seco em estufa com circulação de ar, a $65^{\circ} \mathrm{C}$, até atingir peso constante. O material seco foi moído em um moinho do tipo Wiley. As determinações dos teores macronutrientes, N, $\mathrm{P}, \mathrm{K}, \mathrm{Ca}, \mathrm{Mg}$ e $\mathrm{S}, \mathrm{em} \mathrm{g} / \mathrm{kg}$ de matéria seca, foram realizadas de acordo com o procedimento descrito por MALAVOLTA et al. (1989).

As estimativas de parâmetros genéticos para as características quantitativas foram em nível de média de parcelas, baseando-se em VENCOVSKY \& BARRIGA (1992). Foram realizadas análises individuais, em cada um dos testes de progênie, e uma conjunta, envolvendo ambos os experimentos (Tabelas 1 e 2).

Tabela 1 - Esquema de análise de variância individual no teor de nutrientes em cada população de aroeira de Paulo de Faria, envolvendo duas situações de manejo

Table 1 - Individual variance analysis scheme to each nutrient characteristic, in two "Aroeira" populations of Paulo de Faria, in two management systems

\begin{tabular}{lllll}
\hline FV & GL & $\mathrm{QM}$ & $\mathrm{E}(\mathrm{QM})$ & $\mathrm{F}$ \\
\hline Repetição & $r-1$ & $\mathrm{Q}_{1}$ & $\sigma^{2}+f \sigma_{b}^{2}$ & $\mathrm{Q}_{1} / \mathrm{Q}_{3}$ \\
Famílias & $f-1$ & $\mathrm{Q}_{2}$ & $\sigma^{2}+r \sigma_{p}^{2}$ & $\mathrm{Q}_{2} / \mathrm{Q}_{3}$ \\
Erro & $(r-1)(f-1)$ & $\mathrm{Q}_{3}$ & $\sigma^{2}$ &
\end{tabular}

$\overline{\mathrm{QM}}=$ quadrado médio; $\mathrm{E}(\mathrm{QM})$ = esperança do quadrado médio; $\mathrm{r}=$ número de repetições; $\mathrm{f}=$ número de progênies (famílias); $\sigma_{p}^{2}=$ variância entre progênies; $\sigma^{2}=$ variância do erro entre parcelas. 
Tabela 2 - Esquema da análise de variância conjunta, utilizada na análise de cada uma dos caracteres quantitativos na população de aroeira de Paulo de Faria, envolvendo duas situações de manejo, tendo como fonte de variação: repetições dentro de experimentos $(\mathrm{R} / \mathrm{E})$, experimentos $(\mathrm{E})$, famílias $(\mathrm{F})$, interação entre experimentos e família $(\mathrm{E} \times \mathrm{F})$ e o erro médio

Table 2 - Whole variance scheme to each quantitative characteristic of the Aroeira population Paulo de Faria, in two management systems. It had as variation source: replication into experiment $(R / E)$, experiment $(E)$, family $(F)$, interaction between experiment and family $(\mathrm{ExF})$ and mean error

\begin{tabular}{|c|c|c|c|c|}
\hline $\mathrm{FV}$ & GL & $\mathrm{QM}$ & $\mathrm{E}(\mathrm{QM})$ & $\mathrm{F}$ \\
\hline REP(EXP) & $e(r-1)$ & $\mathrm{Q}_{1}$ & $\sigma_{e}^{2}+f \sigma_{R}^{2}$ & $\mathrm{Q}_{1} / \mathrm{Q}_{5}$ \\
\hline $\operatorname{EXP}(E)$ & $(e-1)$ & $\mathrm{Q}_{2}$ & $\sigma_{e}^{2}+f \sigma_{R}^{2}+r\left(\frac{e}{e-1}\right) \sigma_{F E}^{2}+r f V_{e}$ & $\left(\mathrm{Q}_{2}+\mathrm{Q}_{5}\right) /\left(\mathrm{Q}_{1}+\mathrm{Q}_{4}\right)$ \\
\hline $\mathrm{FAM}(\mathrm{F})$ & $(f-1)$ & $\mathrm{Q}_{3}$ & $\sigma_{e}^{2}+e r \sigma_{F}^{2}$ & $\mathrm{Q}_{3} / \mathrm{Q}_{5}$ \\
\hline$E \times F$ & $(e-1)(f-1)$ & $\mathrm{Q}_{4}$ & $\sigma_{e}^{2}+r\left(\frac{e}{e-1}\right) \sigma_{F E}^{2}$ & $\mathrm{Q}_{4} / \mathrm{Q}_{5}$ \\
\hline $\begin{array}{l}\text { ERRO } \\
\text { MÉDIO }\end{array}$ & $e(r-1)(f-1)$ & $\mathrm{Q}_{5}$ & $\sigma_{e}^{2}$ & - \\
\hline
\end{tabular}

\section{RESULTADOS E DISCUSSÃO}

No experimento em consórcio (EXP1) foram detectadas diferenças significativas entre as médias das progênies nos teores de: N, Ca eb S (Tabela 3). No experimento a pleno sol (EXP2), as diferenças entre as médias das progênies foram significativas somente nos teores de $\mathrm{N}$.

O coeficiente de variação experimental variou de $5,78 \%$ (N) a $22,86 \%$ (P) no $\operatorname{Exp} 1$ e de 4,21\%(N) a 28,67\% (P) no Exp 2, apresentando assim uma maior amplitude de variação no $\operatorname{Exp} 2$, refletindo o fato de que a competição intraespecífica, no caso da aroeira, leva à ocorrência de um maior erro experimental que a competição interespecífica. Porém, as características estudadas tiveram praticamente o mesmo comportamento em ambos os experimentos.

Em relação à média geral das características (Tabela 3), as mesmas tiveram comportamento semelhante nos dois experimentos, exceto para $\mathrm{Ca}$ e $\mathrm{Mg}$, onde os teores no consórcio foram ligeiramente superiores ao do povoamento homogêneo. Na média do povoamento homogêneo verificaram-se dados um pouco menores para $\mathrm{N}$ e $\mathrm{S}$, no presente ensaio, quando comparado aos trabalhos de BARBOSA (1994) e MENDONÇA et al. (1998). Entretanto, estes elementos foram encontrados na mesma magnitude quando comparados aos obtidos por MORAES (1992). Os outros macronutrientes apresentaram médias semelhantes para P, K e Mg às encontradas por FREITAS (1999). Já 
para Ca os dados obtidos estiveram próximos aos de BARBOSA (1994) e MENDONÇA et al. (1998). Esta variação, como já mencionada, depende da idade da folha amostrada, tipo de condução do povoamento, solo, clima, etc. os quais participaram com maior ou menor intensidade na concentração dos elementos nas folhas.

Tabela 3 - Estimativa de parâmetros estatísticos das características nutricionais, aos 26 meses de idade, da população de aroeira de Paulo de Faria - SP, nos experimentos consorciado e homogêneo, em Selvíria - MS

Table 3 - Estimates some statistical parameters to nutrient characteristics to 26 month age trees to Aroeira population of Paulo de Faria -SP, on heterogeneous and homogeneous experiments, in Selvíria - MS

\begin{tabular}{lcll|ccc}
\hline & \multicolumn{5}{c}{ Experimentos } \\
\cline { 2 - 7 } Caráter & \multicolumn{4}{c|}{ Consórcio (1) } & \multicolumn{3}{c}{ Homogêneo (2) } \\
\cline { 2 - 7 } & $\hat{m}$ & \multicolumn{1}{c}{$\mathrm{F}$} & $\mathrm{CV}_{\exp }(\%)$ & $\hat{m}$ & $\mathrm{~F}$ & $\mathrm{CV}_{\text {exp }}(\%)$ \\
\hline $\mathrm{N}(\mathrm{g} / \mathrm{kg})$ & 19,24 & $2,68^{* *}$ & 5,78 & 20,45 & $1,81^{*}$ & 4,21 \\
$\mathrm{P}(\mathrm{g} / \mathrm{kg})$ & 1,92 & 1,59 & 22,86 & 1,46 & 1,55 & 28,67 \\
$\mathrm{~K}(\mathrm{~g} / \mathrm{kg})$ & 12,15 & 1,55 & 20,53 & 11,33 & 1,41 & 12,56 \\
$\mathrm{Ca}(\mathrm{g} / \mathrm{kg})$ & 12,73 & $1,68^{*}$ & 19,35 & 10,06 & 1,15 & 22,67 \\
$\mathrm{Mg}(\mathrm{g} / \mathrm{kg})$ & 3,88 & 1,05 & 19,18 & 2,81 & 1,32 & 16,07 \\
$\mathrm{~S}(\mathrm{~g} / \mathrm{kg})$ & 0,86 & $1,78^{*}$ & 11,97 & 0,93 & 1,09 & 9,91 \\
\hline
\end{tabular}

$\mathrm{CV}_{\text {exp }}$ - coeficiente de variação experimental; $\hat{m}$ - estimativa da média geral; **, * - teste de $\mathrm{F}$ significativo em nível de $1 \%$ e $5 \%$, respectivamente.

Pela análise conjunta dos experimentos (Tabela 4) houve diferença significativa entre os mesmos quanto aos teores de $\mathrm{P}$ e $\mathrm{Ca}$, sem efeito significativo da interação experimento $\mathrm{x}$ família (exceto para $\mathrm{P}$ ). Segundo ALLARD (1971), quando não há interação genótipo ambiente, significa que os genótipos tiveram o mesmo comportamento em todos ambientes. REZENDE (1999), no entanto, considera que a estabilidade média do grupo, provavelmente, advém da interação compensatória dos diferentes indivíduos que compõem o grupo, em relação aos diversos ambientes, contribuindo pouco para informar a respeito da estabilidade de um indivíduo, em particular da família. 
Tabela 4 - Estimativas dos valores de F para os efeitos de experimentos (EXP) e da interação experimento $\mathrm{x}$ família $(\mathrm{E} \times \mathrm{F})$, na análise conjunta, dos experimentos de aroeira, aos 26 meses de idade, em Selvíria - MS

Table 4 - Estimates of $F$ values to experiment effect (ExP) and of interaction to experiment versus family (ExF), on whole analysis, to Aroeira experiment, 26 month age trees, in Selvíria - MS

\begin{tabular}{llll}
\hline Caráter & F.V. & & \\
\cline { 2 - 4 } & EXP & FAM & E x F \\
\hline $\mathrm{N}(\mathrm{g} / \mathrm{kg})$ & 0,55 & $1,65^{*}$ & 0,82 \\
$\mathrm{P}(\mathrm{g} / \mathrm{kg})$ & $21,41^{* *}$ & 1,41 & $1,73^{*}$ \\
$\mathrm{~K}(\mathrm{~g} / \mathrm{kg})$ & 0,27 & $1,69^{*}$ & 1,34 \\
$\mathrm{Ca}(\mathrm{g} / \mathrm{kg})$ & $11,66^{* *}$ & $1,67^{*}$ & 1,20 \\
$\mathrm{Mg}(\mathrm{g} / \mathrm{kg})$ & 2,63 & 1,47 & 0,78 \\
$\mathrm{~S}(\mathrm{~g} / \mathrm{kg})$ & 2,83 & 1,55 & 1,39 \\
\hline
\end{tabular}

**, * - teste de $\mathrm{F}$ significativo em nível de $1 \%$ e $5 \%$ de probabilidade, respectivamente.

Os coeficientes de variação genética $\left(\mathrm{CV}_{\mathrm{g}}\right)$ variaram de $2,51 \%$ a $10,13 \%$, respectivamente, para os teores de $\mathrm{Mg}$ e $\mathrm{P}$ no experimento 1 (Tabela 5). Já, no experimento 2 , os resultados variaram de $1,70 \%$ (S) a $12,28 \%$ (P). Verifica-se que, para o teor de $\mathrm{P}$, as progênies apresentaram maior variação genética, quando comparando o $\mathrm{Ca}, \mathrm{K}$ e o $\mathrm{N}$, em ambos os experimentos, mas ocorreu uma inversão de valores no que se refere ao $\mathrm{Mg}$ e $\mathrm{S}$, sendo que, no experimento consorciado, o $\mathrm{Mg}$ expressou a menor variação genética e, no homogêneo, foi o $\mathrm{S}$. Estes valores de coeficiente de variação genética podem ser comparados com a maioria das espécies nativas estudadas por SIQUEIRA et al. (1998b), KAGEYAMA et al. (1993).

Tabela 5 - Estimativa de alguns parâmetros genéticos para as características nutricionais, aos 26 meses de idade, para a população de aroeira de Paulo de Faria - SP, nos experimentos 1 e 2, em Selvíria - MS

Table 5 - Estimates some genetic parameters to nutrient characteristics, 26 month age tree, to Aroeira population of Paulo de Faria $-S P$, on $1^{\text {st }}$ and $2^{\text {nd }}$ experiments in Selvíria $-M S$

\begin{tabular}{llccc|cccc}
\hline & \multicolumn{6}{c}{ Experimentos } \\
\cline { 2 - 8 } \multicolumn{1}{c}{ Caráter } & \multicolumn{9}{c}{ Consórcio (1) } \\
\cline { 2 - 8 } & $\mathrm{CV}_{\mathrm{g}}$ & $\hat{b}$ & $\hat{h}_{m}^{2}$ & $\mathrm{~s}\left(\hat{h}_{m}^{2}\right)$ & \multicolumn{1}{c}{$\mathrm{CV}_{\mathrm{g}}$} & $\hat{b}$ & $\hat{h}_{m}^{2}$ & $\mathrm{~s}\left(\hat{h}_{m}^{2}\right)$ \\
\hline $\mathrm{N}(\mathrm{g} / \mathrm{kg})$ & 4,32 & 0,75 & 0,63 & 0,1169 & 2,19 & 0,52 & 0,45 & 0,1728 \\
$\mathrm{P}(\mathrm{g} / \mathrm{kg})$ & 10,13 & 0,44 & 0,37 & 0,1969 & 12,28 & 0,43 & 0,35 & 0,2017 \\
$\mathrm{~K}(\mathrm{~g} / \mathrm{kg})$ & 8,79 & 0,43 & 0,35 & 0,2019 & 4,64 & 0,37 & 0,29 & 0,2218 \\
$\mathrm{Ca}(\mathrm{g} / \mathrm{kg})$ & 9,24 & 0,48 & 0,41 & 0,1858 & 5,09 & 0,22 & 0,13 & 0,2717 \\
$\mathrm{Mg}(\mathrm{g} / \mathrm{kg})$ & 2,51 & 0,13 & 0,05 & 0,2975 & 5,25 & 0,33 & 0,24 & 0,2369 \\
$\mathrm{~S}(\mathrm{~g} / \mathrm{kg})$ & 6,11 & 0,51 & 0,44 & 0,1757 & 1,70 & 0,17 & 0,08 & 0,2874 \\
\hline
\end{tabular}


As estimativas de herdabilidade em nível de média $\left(\hat{h}_{m}^{2}\right)$, no experimento 1 (Tabela 5) foram baixas para o conteúdo de $\mathrm{Mg}(0,05)$ e médias para as demais características. No experimento 2, este valor foi baixo para Ca e $\mathrm{S}$ e médio para as outras características. Portanto, a maior herdabilidade foi encontrada para o teor de $\mathrm{N}$, quando comparado a do conteúdo dos demais nutrientes nos dois experimentos, sendo que ocorreu inversão nos valores para $\mathrm{P}, \mathrm{K}, \mathrm{Ca}, \mathrm{Mg}$ e $\mathrm{S}$, onde o $\mathrm{Mg}$ apresentou menor valor no experimento consorciado e o $\mathrm{S}$ no homogêneo. Estes resultados são comparáveis a algumas espécies nativas citadas por SIQUEIRA et al. (1998a), onde estes foram baixo $(0,11)$ ou médio $(0,44)$.

O "índice de seleção" $(\hat{b})$, no experimento 1 , teve valores baixos $(0,13$ para $\mathrm{Mg})$ a altos $(0,75$ para $\mathrm{N})$ ( Tabela 5). No experimento 2, este índice foi baixo (Ca e S) e médio ( $\mathrm{N}, \mathrm{P}, \mathrm{K}$ e $\mathrm{Mg}$ ). Segundo VENCOVSKY \& BARRIGA (1992), este índice indica o potencial da característica para seleção. Assim, o conteúdo de $\mathrm{N}$ foi a característica que apresentou o maior índice de seleção (0,75 - Exp 1 e 0,45 - Exp 2), o que indica que a seleção com base no teor deste nutriente seria mais efetiva que nos demais (P, K, Ca, Mg e $\mathrm{S}$ ).

Os altos valores de $\mathrm{CV}_{\mathrm{g}}$ (para $\left.\mathrm{P}\right)$ e $\hat{h}_{m}^{2}$ (para $\mathrm{N}$ ) encontrados nos experimentos de aroeira indicam a diversidade destas plantas, e assim, boas possibilidades de tolerância a este tipo de solo, normalmente pobres em $\mathrm{P}$ e em matéria orgânica, explicando então, a grande ocorrência desta espécie nestes solos.

A concentração de $\mathrm{Mg}$ e $\mathrm{S}$ depende da quantidade de luz disponível para as plantas, isto é, quanto maior a quantidade de luz disponível maior é a concentração de $\mathrm{Mg}$ e menor de $\mathrm{S}$, ou vice-versa. Desta maneira, pode-se afirmar que a quantidade de luz exerce influência sobre a quantidade de clorofila nas plantas e, conseqüentemente, na eficiência fotossintética. Entretanto, isto não foi confirmado no presente estudo, já que a concentração de $\mathrm{Mg}$ foi um pouco inferior quando no povoamento em consórcio $(3,88 \mathrm{~g} / \mathrm{kg}$ ) comparado ao povoamento homogêneo $(2,81 \mathrm{~g} / \mathrm{kg})$. Para o conteúdo de $\mathrm{S}$ as médias estiveram próximas, sendo de 0,86 e $0,93 \mathrm{~g} / \mathrm{kg}$, no consórcio e no povoamento homogêneo, respectivamente. Isto pode estar associado ao fato dos experimentos ainda estarem no segundo ano de desenvolvimento e que outros fatores, tais como: idade das folhas amostradas, clima, solo, etc. também sejam atuantes.

As correlações genéticas aditivas $\left(r_{A}\right)$, no experimento 1 (Tabela 6), foram altas para as combinações dos teores de $\mathrm{N}$ com $\mathrm{Mg}$ e $\mathrm{S} ; \mathrm{P}$ com $\mathrm{Ca} ; \mathrm{K}$ com $\mathrm{Ca}$; $\mathrm{Ca}$ com $\mathrm{Mg}$; $\mathrm{Mg}$ com $\mathrm{S}$; e foi baixa para $\mathrm{N}$ com $\mathrm{K}$, em ambos os experimentos. Também, ocorreu inversão de posições para as combinações de $\mathrm{N}$ com $\mathrm{P}$ e $\mathrm{Ca}$; $\mathrm{P}$ com $\mathrm{S}$; $\mathrm{K}$ com $\mathrm{Mg}$ e $\mathrm{S}$; $\mathrm{Ca}$ com $\mathrm{S}$, os quais apresentaram valores baixos no experimento 1, e alto no experimento 2. Este comportamento 
foi semelhante ao estudo realizado por FREITAS (1999). Salienta-se aqui o fato da presença de valores de correlação genética superiores a 1, 00, sendo que isto é possível de se obter matematicamente, pois, neste caso, o coeficiente é obtido a partir das estimativas de variâncias, oriundas da esperança dos quadrados médios, pelo método dos momentos (BARBIN, 1993). Tal fato deve-se à relação existente entre a covariância aditiva, envolvendo os dois caracteres, ser muito maior que a multiplicação das variâncias aditivas individuais dos caracteres. Neste caso, a maioria dos geneticistas recomenda que estes valores sejam desprezados.

Tabela 6 - Estimativa de correlações genéticas e fenotípicas para as características nutricionais, aos 26 meses de idade, para a população de aroeira de Paulo de Faria - SP, nos experimentos 1 e 2, em Selvíria - MS

Table 6 - Estimates of genetic and phenotypic correlation to nutrient characteristics, 26 month age trees, to Aroeira population of Paulo de Faria $-S P$, on $1^{\text {st }}$ and $2^{\frac{n d}{d}}$ experiments, in Selviria - MS

\begin{tabular}{|c|c|c|c|c|}
\hline \multirow{3}{*}{ Caráter } & \multicolumn{4}{|c|}{ Experimentos } \\
\hline & \multicolumn{2}{|c|}{ Consórcio (1) } & \multicolumn{2}{|c|}{ Homogêneo (2) } \\
\hline & & $r_{\bar{F}}$ & & \\
\hline$\overline{\mathrm{N} \text { vs } \mathrm{P}}$ & $-0,19$ & $-0,05$ & 0,83 & 0,50 \\
\hline $\mathrm{N}$ vs $\mathrm{K}$ & 0,16 & 0,19 & 0,10 & 0,30 \\
\hline $\mathrm{N}$ vs $\mathrm{Ca}$ & $-0,08$ & $-0,18$ & 1,54 & 0,31 \\
\hline $\mathrm{N}$ vs $\mathrm{Mg}$ & 1,84 & 0,31 & 0,81 & 0,26 \\
\hline $\mathrm{N}$ vs $\mathrm{S}$ & 1,04 & 0,82 & 1,51 & 0,54 \\
\hline P vs K & 0,58 & 0,37 & 0,88 & 0,46 \\
\hline $\mathrm{P}$ vs $\mathrm{Ca}$ & 0,73 & 0,18 & 1,03 & 0,65 \\
\hline $\mathrm{P}$ vs $\mathrm{Mg}$ & $-1,29$ & $-0,02$ & 0,43 & 0,30 \\
\hline $\mathrm{P}$ vs $\mathrm{S}$ & 0,05 & 0,05 & 0,91 & 0,25 \\
\hline $\mathrm{K}$ vs $\mathrm{Ca}$ & 0,67 & 0,19 & 1,67 & 0,32 \\
\hline $\mathrm{K}$ vs $\mathrm{Mg}$ & $-0,15$ & 0,07 & 0,66 & 0,20 \\
\hline K vs $\mathrm{S}$ & 0,28 & 0,19 & $-1,13$ & 0,12 \\
\hline $\mathrm{Ca}$ vs $\mathrm{Mg}$ & 2,09 & 0,14 & 0,99 & 0,48 \\
\hline Ca vs $\mathrm{S}$ & 0,12 & $-0,05$ & 1,17 & $-0,07$ \\
\hline Mg vs S & 1,79 & 0,36 & 1,80 & 0,16 \\
\hline
\end{tabular}


As correlações fenotípicas em nível de média $\left(r_{\bar{F}}\right)$ foram baixas para as combinações dos teores de $\mathrm{P}$ com $\mathrm{S} ; \mathrm{K}$ com $\mathrm{Mg}$ e $\mathrm{S}$; $\mathrm{Ca}$ com $\mathrm{S}$, em ambos os experimentos, ocorrendo uma inversão de posição para $\mathrm{P}$ com $\mathrm{Ca}$ com valor baixo no experimento consorciado, e alto no experimento homogêneo. As demais combinações tiveram inversão de posição de alto ou baixo valor para médio, e de intermediário para superior ou inferior. Resultados semelhantes foram encontrados por FREITAS (1999), trabalhando com uma população de aroeira Petrolina - PE.

Segundo VENCOVSKY \& BARRIGA (1992), quando vários caracteres são medidos num mesmo indivíduo é certo que todos eles serão afetados pelas condições ambientais em que este indivíduo se desenvolveu. Assim, as variações de ambiente, num conjunto de plantas, podem provocar correlações entre os caracteres, positivas ou negativas, de natureza unicamente ambiental.

As correlações fenotípicas $\left(r_{\bar{F}}\right)$, em nível de média, apresentaram-se superiores às correlações genéticas aditivas $\left(r_{A}\right)$, para todas as características, exceto para os teores de $\mathrm{N}$ vs $\mathrm{P}$ e $\mathrm{N}$ vs $\mathrm{K}$, para as quais ocorreu o inverso (tabela 6). Portanto, nos dois experimentos, o efeito genético foi maior que o ambiental, sendo mais acentuado no experimento de aroeira a pleno sol.

\section{CONCLUSÕES}

- As características estudadas apresentaram um comportamento diferenciado quanto à competição inter e intraespecífica;

- Entre os caracteres nutricionais, o conteúdo de nitrogênio foi a característica mais indicada para um processo de seleção;

- O teor de fósforo apresentou maior variabilidade genética em comparação com os demais nutrientes;

- O estudo das características nutricionais permitiu verificar, de modo indireto, o comportamento de uma população de aroeira em plantio consorciado e homogêneo, em relação à absorção de nutrientes, em um solo anteriormente sob vegetação de cerrado.

\section{BIBLIOGRAFIA CITADA}

ALLARD, R.W. 1971. Princípios de melhoramento genético das plantas. São Paulo: Edgard Blücher. 381p. 
ARYA, S; BISHT, R.P.; TOMAR, R.; TOKY, O.P. \& HARRIS, P.J.C. 1995. Genetic variation in minerals, crude protein and structural carbohydrates of foliage in provenances of young plants of Prosopis cineraria (1.) Druce in Índia. Agroforetry Systems, 29:1-7.

BARBIN, D. 1993. Componente de variância: teoria e aplicações. 2. ed.ver. e amp. Piracicaba: FEALQ/ESALQ/USP. 120p.

BARBOSA, Z. 1994. Efeito do fósforo e do zinco na nutrição e crescimento de Myracrodruon urundeuva Fr. All. (aroeira-do-sertão). Lavras. 105p. Dissertação (Mestre em Agronomia) - Escola Superior de Agricultura de Lavras.

DIAS, L.A.S.D. \& KAGEYAMA, P.Y. 1991. Variação genética em espécies arbóreas e conseqüências para o melhoramento florestal. Agrotrópica, 3:119-27.

FREITAS, M.L.M. 1999. Variação genética em progênies de aroeira (Myracrodruon urundeuva Fr. All.) - Anacardiaceae - em diferentes sistemas de plantio. Ilha Solteira. 95p. Dissertação (Mestre em Agronomia) - Faculdade de Engenharia de Ilha Solteira, Universidade Estadual Paulista.

KAGEYAMA, P.Y.; GERES, W.L.A.; ANTIQUEIRA, L.R.; KANO, N.K. \& DIAS, J.H.P. 1993. Teste de progênie combinado de espécies pioneiras e climácicas. In: CONGRESSO FLORESTAL BRASILEIRO, 7, Curitiba. Anais. Curitiba: SBS/SBEF. p.473-5.

MALAVOLTA, E.; VITTI, G.C. \& OLIVEIRA, S.A. 1989. Avaliação do estado nutricional das plantas: princípios e aplicações. Piracicaba: Associação Brasileira para Pesquisa da potassa e do Fosfato, 1989. 195p.

MENDONÇA, A.V.R.; VENTURIN, R.P.; CARVALHO, J.G. \& BASTOS, A. R.R. 1998. Teores foliares de mudas de aroeira em função da relação Ca:Mg (Myracrodruon urundeuva).In: FERTBIO. Caxambu. Anais. p.366.

MORAES, M.L.T. 1992. Variabilidade genética por isoenzimas e caracteres quantitativos em duas populações naturais de aroeira Myracrodruon urundeuva F.F. \& M.F. Allemão - Anacardiaceae (Syn: Astronium urundeuva (Fr. Allemão) Engler). Piracicaba. 139p. Tese (Doutor em Genética e Melhoramento de Plantas) - Escola Superior de Agricultura "Luiz de Queiroz”, Universidade de São Paulo. 
REZENDE, M.D.V. 1999. Melhoramento de essências florestais. In: BORÉM, A. (ed.) Melhoramento de espécies cultivadas. Viçosa: UFV. p.589-647.

SIQUEIRA, A.C.M.F.; GARRIDO, L.M.A.G. \& SEBBENN, A.M. 1998. Interação progênies $\mathrm{x}$ locais e variabilidade genética em jequitibá-rosa Cariniana legalis (Mart.) O. Ktze. Revista do Instituto Florestal, no prelo. (a)

SIQUEIRA, A.C.M.F.; SEBBEN, A.M.; KAGEYAMA, P.Y.; CASTOLLEN, M.S. \& ZANATTO, A.C.S. 1998. Comportamento silvicultural e genético de duas espécies arbóreas tropicais secundárias. Revista do Instituto Florestal, no prelo. (b)

VENCOVSKY, R. \& BARRIGA, P. 1992. Genética biométrica no fitomelhoramento. Ribeirão Preto: Revista Brasileira de Genética. 486p.

\section{AGRADECIMENTOS}

Os autores agradecem aos pesquisadores do Instituto Florestal: Raul O. de Castro, José E. A. Bertoni e Ana Cristina M. F. Siqueira e ao Prof. Dr. Paulo Yoshio Kageyama pelo apoio quando da coleta de sementes na Estação Ecológica de Paulo de Faria - SP. 